\title{
Asymptotic Size Ramsey Results for Bipartite Graphs
}

\author{
Oleg Pikhurko* \\ DPMMS, Centre for Mathematical Sciences \\ Cambridge University, Cambridge CB3 0WB, England \\ E-mail: 0.Pikhurko@dpmms.cam.ac.uk
}

November 1, 2018

\begin{abstract}
We show that $\lim _{n \rightarrow \infty} \hat{r}\left(F_{1, n}, \ldots, F_{q, n}, F_{p+1}, \ldots, F_{r}\right) / n$ exists, where the bipartite graphs $F_{q+1}, \ldots, F_{r}$ do not depend on $n$ while, for $1 \leq i \leq q, F_{i, n}$ is obtained from some bipartite graph $F_{i}$ with parts $V_{1} \cup V_{2}=V\left(F_{i}\right)$ by duplicating each vertex $v \in V_{2}$ $\left(c_{v}+o(1)\right) n$ times for some real $c_{v}>0$.

In fact, the limit is the minimum of a certain mixed integer program. Using the Farkas Lemma we compute it when each forbidden graph is a complete bipartite graph, in particular answering a question of Erdős, Faudree, Rousseau and Schelp (1978) who asked for the asymptotics of $\hat{r}\left(K_{s, n}, K_{s, n}\right)$ for fixed $s$ and large $n$. Furthermore, we prove (for all sufficiently large $n$ ) the conjecture of Faudree, Rousseau and Sheehan (1983) that $\hat{r}\left(K_{2, n}, K_{2, n}\right)=18 n-15$.
\end{abstract}

\section{Introduction}

Let $\left(F_{1}, \ldots, F_{r}\right)$ be an $r$-tuple of graphs which are called forbidden. We say that a graph $G$ arrows $\left(F_{1}, \ldots, F_{r}\right)$ if for any $r$-colouring of $E(G)$, the edge set of $G$, there is a copy of $F_{i}$ of colour $i$ for some $i \in[r]:=\{1, \ldots, r\}$. We denote this arrowing property by $G \rightarrow\left(F_{1}, \ldots, F_{r}\right)$.

The (ordinary) Ramsey number asks for the minimum order of such $G$. Here, however, we deal exclusively with the size Ramsey number

$$
\hat{r}\left(F_{1}, \ldots, F_{r}\right)=\min \left\{e(G) \mid G \rightarrow\left(F_{1}, \ldots, F_{r}\right)\right\}
$$

which is the smallest number of edges that an arrowing graph can have.

Size Ramsey numbers seem hard to compute, even for simple forbidden graphs. For example, the old conjecture of Erdös [7] that $\hat{r}\left(K_{1, n}, K_{3}\right)=3 n(n+1) / 2$ has only recently been disproved in [16], where it is shown that $\hat{r}\left(K_{1, n}, F\right)=(1+o(1)) n^{2}$ for any fixed 3chromatic graph $F$. (Here, $K_{m, n}$ is the complete bipartite graph with parts of sizes $m$ and $n ; K_{n}$ is the complete graph of order $n$.)

*Supported by a Research Fellowship, St. John's College, Cambridge. Part of this research was carried out during the author's stay at the Humboldt University, Berlin, sponsored by the German Academic Exchange Service (DAAD). 
This research initiated as an attempt to find the asymptotics of $\hat{r}\left(K_{1, n}, F\right)$ for a fixed graph $F$. The case $\chi(F) \geq 4$ is treated in [17] (and [16] deals with $\chi(F)=3$ ). What can be said if $F$ is a bipartite graph?

Faudree, Rousseau and Sheehan [12] proved that

$$
\hat{r}\left(K_{1, n}, K_{2, m}\right)=4 n+2 m-4
$$

for every $m \geq 9$ if $n$ is sufficiently large (depending on $m$ ) and stated that their method shows that $\hat{r}\left(K_{1, n}, K_{2,2}\right)=4 n$. They also observed that $K_{s, 2 n}$ arrows the pair $\left(K_{1, n}, C_{2 s}\right)$, where $C_{2 s}$ is a cycle of order $2 s$; hence $\hat{r}\left(K_{1, n}, C_{2 s}\right) \leq 2 s n$.

Let $P_{s}$ be the path with $s$ vertices. Lortz and Mengersen 15 showed that $K_{k, 2 n-1} \rightarrow$ $\left(K_{1, n}, P_{2 k+1}\right)$ and $K_{k}+\bar{K}_{2 n-k-1} \rightarrow\left(K_{1, n}, P_{2 k}\right)$ and conjectured that this is sharp for any $s \geq 4$ provided $n$ is sufficiently large, that is,

$$
\hat{r}\left(K_{1, n}, P_{s}\right)=\left\{\begin{array}{ll}
2 k n-k, & \text { if } s=2 k+1, \\
2 k n-k(k+3) / 2, & \text { if } s=2 k,
\end{array} \quad n \geq n_{0}(s) .\right.
$$

The conjecture was proved for $4 \leq s \leq 7$ in 15$]$.

Size Ramsey numbers $\hat{r}\left(F_{1}, F_{2}\right)$ for bipartite graphs $F_{1}$ and $F_{2}$ (and in some papers $F_{1}$ is a small star) are also studied in 10, 6, 3, 4, 9, 11, 14, 13 for example.

It is not hard to see that, for fixed $s_{1}, \ldots, s_{r} \in \mathbb{N}$ and $t_{1}, \ldots, t_{r} \in \mathbb{R}_{>0}$, we have

$$
\hat{r}\left(K_{s_{1},\left\lfloor t_{1} n\right\rfloor}, \ldots, K_{s_{r},\left\lfloor t_{r} n\right\rfloor}\right)=O(n) .
$$

This follows, for example, by assuming that $s_{1}=\ldots=s_{r}=s, t_{1}=\ldots=t_{r}=t$ and considering $K_{v_{1}, v_{2}}$, where $v_{1}=(s-1) r+1$ and $v_{2}=\left\lceil r t n\left(\begin{array}{c}v_{1} \\ s\end{array}\right)\right\rceil$. The latter graph has the required arrowing property. Indeed, for any $r$-colouring, each vertex of $V_{2}$ is incident to at least $s$ edges of same colour; hence there are at least $v_{2}$ monochromatic $K_{s, 1}$-subgraphs and some $S \in\left(\begin{array}{c}V_{1} \\ s\end{array}\right)$ appears in at least $r$ tn such subgraphs of which at least $t n$ have same colour.

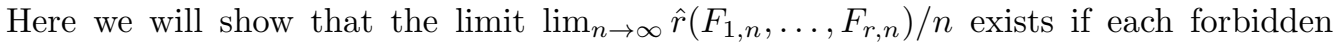
graph is either a fixed bipartite graph or a subgraph of $K_{s,\lfloor t n\rfloor}$ which 'dilates' uniformly with $n$ (the precise definition will be given in Section 2). In particular, $\hat{r}\left(K_{1, n}, F\right) / n$ tends to a limit for any fixed bipartite graph $F$.

The limit value can in fact be obtained as the minimum of a certain mixed integer program (which does depend on $n$ ). We have been able to solve the MIP when each $F_{i, n}$ is a complete bipartite graph. In particular, this answers a question by Erdős, Faudree, Rousseau and Schelp [10, Problem B] who asked for the asymptotics of $\hat{r}\left(K_{s, n}, K_{s, n}\right)$. Working harder on the case $s=2$ we prove (for all sufficiently large $n$ ) the conjecture of Faudree, Rousseau and Sheehan [12, Conjecture 15] that

$$
\hat{r}\left(K_{2, n}, K_{2, n}\right)=18 n-15,
$$

where the upper bound is obtained by considering $K_{3,6 n-5} \rightarrow\left(K_{2, n}, K_{2, n}\right)$. Unfortunately, the range on $n$ from (3) is not specified in [12], although it is stated there that $\hat{r}\left(K_{2,2}, K_{2,2}\right)=$ 15 , where the upper bound follows apparently from $K_{6} \rightarrow\left(K_{2,2}, K_{2,2}\right)$.

Unfortunately, our MIP is not well suited for practical calculations and we were not able to compute the asymptotics for any other non-trivial forbidden graphs; in particular, 
we had no progress on (11). But we hope that the introduced method will produce more results: although the MIP is hard to solve, it may well be possible that, for example, some manageable relaxation of it gives good lower or upper bounds.

Our method does not work if we allow both vertex classes of forbidden graphs to grow with $n$. In these settings, in fact, we do not know the asymptotics even in simplest cases. For example, the best known bounds on $r=\hat{r}\left(K_{n, n}, K_{n, n}\right)$ seem to be $r<\frac{3}{2} n^{3} 2^{n}$ for $n \geq 6$ (Erdős, Faudree, Rousseau and Schelp [10]) and $r>\frac{1}{60} n^{2} 2^{n}$ (Erdős and Rousseau [1]).

Our theorem on the existence of the limit can be extended to generalized size Ramsey problems; this is discussed in Section 5 .

\section{Some Definitions}

We decided to gather most of the definitions in this section for quick reference.

We assume that bipartite graphs come equipped with a fixed bipartition $V(F)=V_{1}(F) \cup$ $V_{2}(F)$, although graph embeddings need not preserve it. We denote $v_{i}(F)=\left|V_{i}(F)\right|, i=1,2$; thus $v(F)=v_{1}(F)+v_{2}(F)$.

For $A \subset V_{1}(F)$, we define

$$
F^{A}=\left\{v \in V_{2}(F) \mid \Gamma_{F}(v)=A\right\},
$$

where $\Gamma_{F}(v)$ denotes the neighbourhood of $v$ in $F$. (We will write $\Gamma(v)$, etc., when the encompassing graph $F$ is clear from the context.) Clearly, in order to determine $F$ (up to an isomorphism) it is enough to have $V_{1}(F)$ and $\left|F^{A}\right|$ for all $A \subset V_{1}(F)$. This motivates the following definitions.

A weight $\mathbf{f}$ on a set $V(\mathbf{f})$ a sequence $\left(f_{A}\right)_{A \in 2^{V(\mathbf{f})}}$ of non-negative reals. A bipartite graph $F$ agrees with $\mathbf{f}$ if $V_{1}(F)=V(\mathbf{f})$ and $F^{A}=\emptyset$ if and only if $f_{A}=0, A \in 2^{V(\mathbf{f})}$. A sequence of bipartite graphs $\left(F_{n}\right)_{n \in \mathbb{N}}$ is a dilatation of $\mathbf{f}$ (or dilates $\mathbf{f}$ ) if each $F_{n}$ agrees with $\mathbf{f}$ and

$$
\left|F_{n}^{A}\right|=f_{A} n+o(n), \quad \text { for all } A \in 2^{V(\mathbf{f})} .
$$

(Of course, the latter condition is automatically true for all $A \in 2^{V(\mathbf{f})}$ with $f_{A}=0$.) Clearly, $e\left(F_{n}\right)=(e(\mathbf{f})+o(1)) n$, where $e(\mathbf{f})=\sum_{A \in 2^{V(\mathbf{f})}} f_{A}|A|$, so we call $e(\mathbf{f})$ the size of $\mathbf{f}$. Also, the order of $\mathbf{f}$ is $v(\mathbf{f})=|V(\mathbf{f})|$ and the degree of $x \in V(\mathbf{f})$ is

$$
d(x)=\sum_{\substack{A \in 2 V(\mathbf{f}) \\ A \ni x}} f_{A}
$$

Clearly, $e(\mathbf{f})=\sum_{x \in V(\mathbf{f})} d(x)$.

For example, given $t \in \mathbb{R}_{>0}$, the sequence $\left(K_{s,\lceil t n\rceil}\right)_{n \in \mathbb{N}}$ is the dilatation of $\mathbf{k}_{s, t}$, where the symbol $\mathbf{k}_{s, t}$ will be reserved for the weight on $[s]$ which has value $t$ on $[s]$ and zero otherwise. (We assume that $V_{1}\left(K_{s,[t n\rceil}\right)=[s]$.) It is not hard to see that any sequence of bipartite graphs described in the abstract is in fact a dilatation of some weight.

We write $F \subset \mathbf{f}$ if for some bipartition $V(F)=V_{1}(F) \cup V_{2}(F)$ there is an injection $h: V_{1}(F) \rightarrow V(\mathbf{f})$ such that for any $A \subset V_{1}(F)$ dominated by a vertex of $V_{2}(F)$ there is $B \subset V(\mathbf{f})$ with $h(A) \subset B$ and $f_{B}>0$. This notation is justified by the following trivial lemma. 
Lemma 1 Let $\left(F_{n}\right)_{n \in \mathbb{N}}$ be a dilatation of $\mathbf{f}$. If $F \subset \mathbf{f}$, then $F$ is a subgraph of $F_{n}$ for all sufficiently large $n$. Otherwise, which is denoted by $F \not \subset \mathbf{f}$, no $F_{n}$ contains $F$.

Let $\mathbf{f}$ and $\mathbf{g}$ be weights. Assume that $v(\mathbf{f}) \leq v(\mathbf{g})$ by adding new vertices to $V(\mathbf{g})$ and letting $\mathbf{g}$ be zero on all new sets. We write $\mathbf{f} \subset \mathbf{g}$ if there is an injection $h: V(\mathbf{f}) \rightarrow V(\mathbf{g})$ and numbers $\left(w_{A B} \geq 0\right)_{A \in 2^{V(\mathbf{f})}, B \in 2^{V(\mathbf{g})}}$ such that

$$
\begin{aligned}
\forall A \in 2^{V(\mathbf{f})}, \quad \forall B \in 2^{V(\mathbf{g})} \quad h(A) \not \subset B \Rightarrow w_{A B}=0, \\
\forall A \in 2^{V(\mathbf{f})} \quad \sum_{\substack{B \in 2^{V(\mathbf{g})} \\
B \supset h(A)}} w_{A B} \geq f_{A}, \\
\forall B \in 2^{V(\mathbf{g})} \quad \sum_{\substack{A \in 2^{V(\mathbf{f})} \\
h(A) \subset B}} w_{A B} \leq g_{B} .
\end{aligned}
$$

This can be viewed as a fractional analogue of the subgraph relation $F \subset G$ : $h$ embeds $V_{1}(F)$ into $V_{1}(G)$ and $w_{A B}$ says how much of $F^{A} \subset V_{2}(F)$ is mapped into $G^{B}$. The fractional $\subset$-relation enjoys many properties of the discrete one. For example, $d(x) \leq d(h(x))$ for any $x \in V(\mathbf{f})$ :

$$
d(x)=\sum_{\substack{A \in 2^{V(\mathbf{f})} \\ A \ni x}} f_{A} \leq \sum_{\substack{A \in 2^{2} V(\mathbf{f}) \\ A \ni x}} \sum_{\substack{B \in 2^{\prime} V(\mathbf{g}) \\ B \supset h(A)}} w_{A, B} \leq \sum_{\substack{B \in 2^{2} V(\mathbf{g}) \\ B \ni h(x)}} \sum_{\substack{A \in \in^{2} V(\mathbf{f}) \\ h(A) \subset B}} w_{A, B} \leq \sum_{\substack{B \in 2^{\prime} V(\mathbf{g}) \\ B \ni h(x)}} g_{B}=d(h(x)) .
$$

The following result is not difficult and, in fact, we will implicitly prove a sharper version later (with concrete estimates of $\epsilon$ ), so we omit the proof.

Lemma 2 Let $\left(F_{n}\right)_{n \in \mathbb{N}}$ and $\left(G_{n}\right)_{n \in \mathbb{N}}$ be dilatations of $\mathbf{f}$ and $\mathbf{g}$ respectively. Then $\mathbf{f} \subset \mathbf{g}$ implies that for any $\epsilon>0$ there is $n_{0}$ such that $F_{n} \subset G_{m}$ for any $n \geq n_{0}$ and $m \geq(1+\epsilon) n$. Otherwise, which is denoted by $\mathbf{f} \not \subset \mathbf{g}$, there is $\epsilon>0$ and $n_{0}$ such that $F_{n} \not \subset G_{m}$ for any $n \geq n_{0}$ and $m \leq(1+\epsilon) n$.

An $r$-colouring $\mathbf{c}$ of $\mathbf{g}$ is a sequence $\left(c_{A_{1}, \ldots, A_{r}}\right)$ of non-negative reals indexed by $r$-tuples of disjoint subsets of $V(\mathbf{g})$ such that

$$
\sum_{A_{1} \cup \cdots \cup A_{r}=A} c_{A_{1}, \ldots, A_{r}}>g_{A}, \quad \text { for all } A \in 2^{V(\mathbf{g})} .
$$

The $i$-th colour subweight $\mathbf{c}_{i}$ is defined by $V\left(\mathbf{c}_{i}\right)=V(\mathbf{g})$ and

$$
c_{i, A}=\sum_{\substack{A_{1}, \ldots, A_{r} \\ A_{i}=A}} c_{A_{1}, \ldots, A_{r}}, \quad A \in 2^{V(\mathbf{g})} .
$$

The analogy: to define an $r$-colouring of $G$, it is enough to define, for all disjoint $A_{1}, \ldots, A_{r} \subset$ $V_{1}(G)$, how many vertices of $G^{A_{1} \cup \cdots \cup A_{r}}$ are connected, for all $i \in[r]$, by colour $i$ precisely to $A_{i}$. Following this analogy, there should have been the equality sign in (5); however, the chosen definition will make our calculations less messy later.

\section{Existence of Limit}

Let $r \geq q \geq 1$. Consider a sequence $\mathbf{F}=\left(\mathbf{F}_{1}, \ldots, \mathbf{F}_{r}\right)$, where $\mathbf{F}_{i}=\mathbf{f}_{i}$ is a weight for $i \in[q]$ and $\mathbf{F}_{i}=F_{i}$ is a bipartite graph for $i \in[q+1, r]$. Assume that $\mathbf{F}_{i}$ does not have an isolated 
vertex (that is, $x \in V\left(\mathbf{F}_{i}\right)$ with $d(x)=0$ ), $i \in[r]$. We say that a weight $\mathbf{g}$ arrows $\mathbf{F}$ (denoted by $\mathbf{g} \rightarrow \mathbf{F}$ ) if for any $r$-colouring $\mathbf{c}$ of $\mathbf{g}$ we have $\mathbf{F}_{i} \subset \mathbf{c}_{i}$ for some $i \in[r]$. Define

$$
\hat{r}(\mathbf{F})=\inf \{e(\mathbf{g}) \mid \mathbf{g} \rightarrow \mathbf{F}\} .
$$

The definition (7) imitates that of the size Ramsey number and we will show that these are very closely related indeed. However, we need a few more preliminaries.

Observe that $\hat{r}(\mathbf{F})<\infty$ by considering $\mathbf{k}_{a, b}$ which arrows $\mathbf{F}$ if, for example, $a=1-$ $r+\sum_{i=1}^{r} v\left(\mathbf{F}_{i}\right)$ and $b$ is sufficiently large, cf. (2). Let $l$ be an integer greater than $\hat{r}(\mathbf{F}) / d_{0}$, where $d_{0}=\sum_{i=1}^{q} d_{i}$ and

$$
d_{i}=\min \left\{d_{\mathbf{f}_{i}}(x) \mid x \in V\left(\mathbf{f}_{i}\right)\right\}>0, \quad i \in[q] .
$$

Lemma $3 \hat{r}(\mathbf{F})=\hat{r}_{l}(\mathbf{F})$, where $r_{l}(\mathbf{F})=\min \{e(\mathbf{g}) \mid \mathbf{g} \rightarrow \mathbf{F}, v(\mathbf{g}) \leq l\}$.

Proof. Let $\epsilon>0$ be any real smaller than $d_{0}$. Let $\mathbf{g} \rightarrow \mathbf{F}$ be a weight with $v(\mathbf{g})>l$ and $e(\mathbf{g}) \leq \hat{r}(\mathbf{F})+\epsilon$. To prove the theorem, it is enough to construct $\mathbf{g}^{\prime} \rightarrow \mathbf{F}$ with $e\left(\mathbf{g}^{\prime}\right) \leq e(\mathbf{g})$ and $v\left(\mathbf{g}^{\prime}\right)=v(\mathbf{g})-1$.

We have $d(x) \leq(\hat{r}(\mathbf{F})+\epsilon) /(l+1)<d_{0}$ for some $x \in V(\mathbf{g})$. Choose $\delta>0$ with $\delta+d_{i} d(x) / d_{0}<d_{i}$ for any $i \in[q]$. Define the weight $\mathbf{g}^{\prime}$ on $V(\mathbf{g}) \backslash\{x\}$ by $g_{A}^{\prime}=g_{A}+g_{A \cup\{x\}}$, $A \in 2^{V\left(\mathbf{g}^{\prime}\right)}$. Clearly, $e\left(\mathbf{g}^{\prime}\right)=e(\mathbf{g})-d(x) \leq e(\mathbf{g})$.

We claim that $\mathbf{g}^{\prime}$ arrows $\mathbf{F}$. Suppose that this is not true and let $\mathbf{c}^{\prime}$ be an $\mathbf{F}$-free $r$ colouring of $\mathbf{g}^{\prime}$. We can assume that

$$
\sum_{A_{1} \cup \cdots \cup A_{r}=A} c_{A_{1}, \ldots, A_{r}}^{\prime} \leq g_{A}^{\prime}+\delta / r^{l}, \quad \text { for any } A \in 2^{V\left(\mathbf{g}^{\prime}\right)} .
$$

Define $\mathbf{c}$ by

$$
c_{A_{1}, \ldots, A_{r}}= \begin{cases}\frac{\lambda_{A \backslash\{x\}} d_{i}}{d_{0}} \cdot c_{A_{1}, \ldots, A_{i-1}, A_{i} \backslash\{x\}, A_{i+1}, \ldots, A_{r},}^{\prime}, & x \in A_{i}, i \in[q], \\ 0, & x \in A_{q+1} \cup \ldots \cup A_{r}, \\ \left(1-\lambda_{A}\right) \cdot c_{A_{1}, \ldots, A_{r}}^{\prime}, & x \notin A,\end{cases}
$$

where we denote $A=A_{1} \cup \cdots \cup A_{r}, \lambda_{A}=g_{A \cup\{x\}} / g_{A}^{\prime}$ if $g_{A}^{\prime}>0$, and $\lambda_{A}=1 / 2$ if $g_{A}^{\prime}=0$. The reader can check that $\mathbf{c}$ is an $r$-colouring of $\mathbf{g}$.

By the assumption on $\mathbf{g}$, we have $\mathbf{F}_{i} \subset \mathbf{c}_{i}$ for some $i \in[r]$. But this embedding cannot use $x$ because for $i \in[q+1, r]$ we have $d_{\mathbf{c}_{i}}(x)=0$ while for $i \in[q]$

$$
\begin{aligned}
d_{\mathbf{c}_{i}}(x) & =\sum_{A_{1}, \ldots, A_{r} \subset V\left(\mathbf{g}^{\prime}\right)} c_{A_{1}, \ldots, A_{i-1}, A_{i} \cup\{x\}, A_{i+1}, \ldots, A_{r}}=\sum_{A \in 2^{V\left(\mathbf{g}^{\prime}\right)}} \frac{\lambda_{A} d_{i}}{d_{0}} \sum_{A_{1} \cup \cdots \cup A_{r}=A} c_{A_{1}, \ldots, A_{r}}^{\prime} \\
& \leq \sum_{A \in 2^{V\left(\mathbf{g}^{\prime}\right)}} \frac{\lambda_{A} d_{i}}{d_{0}}\left(g_{A}^{\prime}+\delta / r^{l}\right) \leq \frac{d_{i} \delta}{d_{0}}+\frac{d_{i}}{d_{0}} \sum_{A \in 2^{V\left(\mathbf{g}^{\prime}\right)}} g_{A \cup\{x\}} \leq \delta+d_{i} \frac{d(x)}{d_{0}}<d_{i}
\end{aligned}
$$

is too small, see (位). But $c_{i, A} \leq c_{i, A}^{\prime}$ for $A \in 2^{V\left(\mathbf{g}^{\prime}\right)}$; hence, $\mathbf{F}_{i} \subset \mathbf{c}_{i}^{\prime}$, which is the desired contradiction.

Hence, to compute $\hat{r}(\mathbf{F})$ it is enough to consider $\mathbf{F}$-arrowing weights on $L=[l]$ only. 
Lemma 4 There exists $\mathbf{g} \rightarrow \mathbf{F}$ with $V(\mathbf{g}) \subset L$ and $e(\mathbf{g})=\hat{r}(\mathbf{F})$. (And we call such a weight extremal.)

Proof. Let $\mathbf{g}_{n} \rightarrow \mathbf{F}$ be a sequence with $V\left(\mathbf{g}_{n}\right) \subset L$ such that $e\left(\mathbf{g}_{n}\right)$ approaches $\hat{r}(\mathbf{F})$. By choosing a subsequence, assume that $V\left(\mathbf{g}_{n}\right)$ is constant and $g_{A}=\lim _{n \rightarrow \infty} g_{n, A}$ exists for each $A \in 2^{L}$. Clearly, $e(\mathbf{g})=\hat{r}(\mathbf{F})$ so it remains to show that $\mathbf{g} \rightarrow \mathbf{F}$.

Let $\mathbf{c}$ be an $r$-colouring of $\mathbf{g}$. Let $\delta$ be the smallest slack in inequalities (5). Choose sufficiently large $n$ so that $\left|g_{n, A}-g_{A}\right|<\delta$ for all $A \in 2^{L}$. We have

$$
\sum_{A_{1} \cup \cdots \cup A_{r}=A} g_{A_{1}, \ldots, A_{r}} \geq g_{A}+\delta>g_{n, A}, \quad A \in 2^{L},
$$

that is, $\mathbf{c}$ is a colouring of $\mathbf{g}_{n}$ as well. Hence, $\mathbf{F}_{i} \subset \mathbf{c}_{i}$ for some $i$, as required.

Now we are ready to prove our general theorem. Its proof essentially takes care of itself. We just exploit the parallels between the fractional and discrete universes, which, unfortunately, requires messing around with various constants.

Theorem 5 Let $\left(F_{i, n}\right)_{n \in \mathbb{N}}$ be a dilatation of $\mathbf{f}_{i}, i \in[q]$. Then, for all sufficiently large $n$,

$$
\hat{r}(\mathbf{F}) n-M\left(1+f_{0}\right) \leq \hat{r}\left(F_{1, n}, \ldots, F_{q, n}, F_{q+1}, \ldots, F_{r}\right) \leq \hat{r}(\mathbf{F}) n+M\left(1+f_{0}\right),
$$

where $f_{0}=\max \left\{|| F_{i}^{A}\left|-f_{i, A} n\right| \mid i \in[q], A \in V\left(\mathbf{f}_{i}\right)\right\}$ and $M=M(\mathbf{F})$ is some constant.

In particular, the limit $\lim _{n \rightarrow \infty} \hat{r}\left(F_{1, n}, \ldots, F_{q, n}, F_{q+1}, \ldots, F_{r}\right) / n$ exists.

Proof. Let $v_{0}=\max \left\{v\left(F_{i}\right) \mid i \in[r]\right\}, m_{1}=2^{v_{0}}\left(f_{0}+1\right)$, and $m_{2}=r^{l} m_{1}+1$.

We will prove that

$$
\hat{r}\left(F_{1}, \ldots, F_{r}\right) \leq \hat{r}(\mathbf{F}) n+2^{l} l\left(m_{2}+1\right), \quad n \geq 1 .
$$

By Lemma 4 choose an extremal weight $\mathbf{g}$ on $L$. Define a bipartite graph $G$ as follows. Choose disjoint from each other (and from $L$ ) sets $G^{A}$ with $\left|G^{A}\right|=\left\lceil g_{A} n+m_{2}\right\rceil, A \in 2^{L}$. Let $V(G)=L \cup\left(\cup_{A \in 2^{L}} G^{A}\right)$. In $G$ we connect $x \in L$ to all of $G^{A}$ if $x \in A$. These are all the edges. Clearly,

$$
e(G)=\sum_{A \in 2^{L}}\left|G^{A}\right||A| \leq 2^{l} l\left(m_{2}+1\right)+\sum_{A \in 2^{L}} g_{A} n|A| \leq \hat{r}(\mathbf{F}) n+2^{l} l\left(m_{2}+1\right),
$$

as required. Hence, it is enough to show that $G$ has the arrowing property.

Consider any $r$-colouring $c: E(G) \rightarrow[r]$. For disjoint sets $B_{1}, \ldots, B_{r} \subset L$, let

$$
\begin{aligned}
C_{B_{1}, \ldots, B_{r}} & =\left\{y \in G^{B} \mid \forall i \in[r], \forall x \in B_{i} c(\{x, y\})=i\right\}, \\
c_{B_{1}, \ldots, B_{r}} & = \begin{cases}\left(\left|C_{B_{1}, \ldots, B_{r}}\right|-m_{1}\right) / n, & \text { if }\left|C_{B_{1}, \ldots, B_{r}}\right| \geq m_{1}, \\
0, & \text { otherwise, }\end{cases}
\end{aligned}
$$

where $B=B_{1} \cup \cdots \cup B_{r}$. Clearly, $n c_{B_{1}, \ldots, B_{r}} \geq\left|C_{B_{1}, \ldots, B_{r}}\right|-m_{1}$; hence,

$$
n \sum_{B_{1} \cup \cdots \cup B_{r}=B} c_{B_{1}, \ldots, B_{r}} \geq-r^{|B|} m_{1}+\sum_{B_{1} \cup \cdots \cup B_{r}=B}\left|C_{B_{1}, \ldots, B_{r}}\right| \geq-r^{l} m_{1}+\left|G^{B}\right|>g_{B},
$$

that is, $\mathbf{c}$ is an $r$-colouring of $\mathbf{g}$. Hence, $\mathbf{F}_{i} \subset \mathbf{c}_{i}$ for some $i \in[r]$. 
Suppose that $i \in[q]$. By the definition, we find appropriate $h: V\left(\mathbf{f}_{i}\right) \rightarrow L$ and $\mathbf{w}$. We aim at proving that $F_{i, n} \subset G_{i}$, where $G_{i} \subset G$ is the colour- $i$ subgraph. Partition $F_{i, n}^{A}=\cup_{B \supset h(A)} W_{A, B}$ so that $W_{A, B}=\emptyset$ if $w_{A, B}=0$ and $\left|W_{A, B}\right| \leq\left\lfloor w_{A, B} n+f_{0}+1\right\rfloor$, $A \in 2^{V\left(\mathbf{f}_{i}\right)}, B \in 2^{L}$. This is possible for any $A$ : if $w_{A, B}=0$ for all $B \in 2^{L}$ with $h(A) \subset B$, then $f_{i, A}=0$ and $F^{A}=\emptyset$; if $w_{A, B}>0$ for at least one $B$, then

$$
\sum_{\substack{B \in 2^{L} L \\ w_{A, B}>0}}\left(w_{A, B} n+f_{0}\right) \geq f_{0}+n \sum_{\substack{B \in 2^{L} L \\ w_{A, B}>0}} w_{A, B} \geq f_{0}+f_{i, A} n \geq\left|F_{i, n}^{A}\right| .
$$

Let $B \in 2^{L}$. If $c_{i, B}=0$, then $\left|W_{A, B}\right|=w_{A, B}=0$ for all $A \in 2^{V\left(\mathbf{f}_{i}\right)}$. Otherwise,

$$
c_{i, B} n=n \sum_{\substack{B_{1}, \ldots, B_{r} \\ B_{i}=B}} c_{B_{1}, \ldots, B_{i}} \leq-m_{1}+\sum_{\substack{B_{1}, \ldots, B_{r} \\ B_{i}=B}}\left|C_{B_{1}, \ldots, B_{i}}\right|=\left|G_{i}^{B}\right|-m_{1},
$$

and we have

$$
\sum_{\substack{A \in 2 V\left(F_{i, n}\right) \\ h(A) \subset B}}\left|W_{A, B}\right| \leq \sum_{\substack{A \in 2 V\left(F_{i, n}\right) \\ h(A) \subset B}}\left(w_{A, B} n+f_{0}+1\right) \leq c_{i, B} n+2^{v_{0}}\left(f_{0}+1\right) \leq\left|G_{i}^{B}\right| .
$$

Hence, we can extend $h$ to the whole of $V\left(F_{i, n}\right)$ by mapping $\cup_{h(A) \subset B} W_{A, B}$ injectively into $B$.

Suppose that $i \in[q+1, r]$. The relation $F_{i} \subset \mathbf{c}_{i}$ means that there exist appropriate $V_{1}\left(F_{i}\right) \cup V_{2}\left(F_{i}\right)=V\left(F_{i}\right)$ and $h: V_{1}\left(F_{i}\right) \rightarrow L$. We view $h$ as a partial embedding of $F_{i}$ into $G_{i}$ and will extend $h$ to the whole of $V\left(F_{i}\right)$.

Take consecutively $y \in V_{2}\left(F_{i}\right)$. There is $B_{i} \subset L$ such that $c_{i, B_{i}}>0$ and $h(\Gamma(y)) \subset$ $B_{i}$. The inequality $c_{i, B_{i}}>0$ implies that there are disjoint $B_{j}$ 's, $j \in[r] \backslash\{i\}$, such that $c_{B_{1}, \ldots, B_{r}}>0$. Each vertex in $C_{B_{1}, \ldots, B_{r}}$ is connected by colour $i$ to the whole of $B_{i} \supset h(\Gamma(y))$. The inequality $c_{B_{1}, \ldots, B_{r}}>0$ means that $\left|C_{B_{1}, \ldots, B_{r}}\right| \geq m_{1} \geq v\left(F_{i}\right)$, so we can always extend $h$ to $y$. Hence, we find an $F_{i}$-subgraph of colour $i$ in this case.

Thus the constructed graph $G$ has the desired arrowing property, which proves the upper bound.

Let $m_{3}=\max \left(r^{4 l / d^{\prime}}, 2^{v_{0}} f_{0}\right)$, where $d^{\prime}=\min _{i \in[q]} \min _{x \in V\left(\mathbf{f}_{i}\right)} d_{f_{i}}(x)>0$. As the lower bound, we show that, for all sufficiently large $n$,

$$
\hat{r}\left(F_{1}, \ldots, F_{r}\right) \geq \hat{r}(\mathbf{F}) n-4 l \cdot 2^{4 l / d^{\prime}} \cdot m_{3} / d^{\prime} .
$$

Suppose on the contrary that we can find an arrowing graph $G$ contradicting (10). Let $L \subset V(G)$ be the set of vertices of degree at least $d^{\prime} n / 2$ in $G$. From $d^{\prime} n|L| / 4<e(G)<\ln$ it follows that $|L| \leq 4 l / d^{\prime}$. For $A \in 2^{L}$, define $g_{A}=\left(\left|G^{A}\right|+m_{3}\right) / n$.

We have

$$
\sum_{A \in 2^{L}} g_{A}|A| \leq \frac{2^{4 l / d^{\prime}} m_{3}}{n} \cdot \frac{4 l}{d^{\prime}}+\frac{1}{n} \sum_{A \in 2^{L}}\left|G^{A}\right||A| \leq \frac{4 l \cdot 2^{4 l / d^{\prime}} m_{3} / d^{\prime}+e(G)}{n}<\hat{r}(\mathbf{F}) .
$$

Thus there is an $\mathbf{F}$-free $r$-colouring $\mathbf{c}$ of $\mathbf{g}$. We are going to exhibit a contradictory $r$-colouring of $E(G)$.

For each $B \in 2^{L}$ choose any disjoint sets $C_{B_{1}, \ldots, B_{r}} \subset G^{B}$ (indexed by $r$-tuples of disjoint sets partitioning $B$ ) such that they partition $G^{B}$ and $\left|C_{B_{1}, \ldots, B_{r}}\right| \leq\left\lfloor c_{B_{1}, \ldots, B_{r}} \cdot n\right\rfloor$. This is possible because

$$
\sum_{B_{1} \cup \cdots \cup B_{r}=B}\left\lfloor c_{B_{1}, \ldots, B_{r}} \cdot n\right\rfloor \geq g_{B} n-r^{4 l / d^{\prime}} \geq\left|G^{B}\right| .
$$


For $j \in[r], x \in B_{j}$ and $y \in C_{B_{1}, \ldots, B_{r}}$, colour the edge $\{x, y\}$ by colour $j$. All the remaining edges of $G$ (namely, those lying inside $L$ or inside $V(G) \backslash L$ ) are coloured with colour 1 .

There is $i \in[r]$ such that $G_{i} \subset G$, the colour- $i$ subgraph, contains a forbidden subgraph.

Suppose that $i \in[q]$. Let $h: F_{i, n} \rightarrow G_{i}$ be an embedding. If $n$ is large, then $d(x)>$ $4 l / d^{\prime}+d^{\prime} n / 2$ for all $x \in V_{1}\left(F_{i, n}\right)$, which implies that $h\left(V_{1}\left(F_{i, n}\right)\right) \subset L$. Define for $A \in 2^{V\left(\mathbf{f}_{i}\right)}$ and $B \in 2^{L}$ with $B \supset h(A)$ and $f_{i, A} \neq 0$

$$
w_{A, B}=\frac{\left|h^{-1}\left(G^{B}\right) \cap F_{i}^{A}\right|+f_{0}}{n} .
$$

All other $w_{A, B}$ 's are set to zero. For $A \in 2^{V\left(\mathbf{f}_{i}\right)}$ with $f_{i, A} \neq 0$, we have

$$
\sum_{\substack{B \in 2 L \\ B \supset h(A)}} w_{A, B} \geq\left(\left|F_{i}^{A}\right|+f_{0}\right) / n \geq f_{i, A}
$$

For $B \in 2^{L}$ we have

$$
\sum_{\substack{A \in 2^{V}\left(\mathbf{f}_{i}\right) \\ h(A) \subset B}} w_{A, B} \leq \frac{2^{v_{0}} f_{0}}{n}+\sum_{\substack{A \in \varepsilon^{2} V\left(\mathfrak{f}_{i}\right) \\ h(A) \subset B}} \frac{\left|h^{-1}\left(G^{B}\right) \cap F_{i}^{A}\right|}{n} \leq \frac{2^{v_{0}} f_{0}}{n}+\frac{\left|G^{B}\right|}{n} \leq g_{B},
$$

that is, $h$ (when restricted to $V\left(\mathbf{f}_{i}\right)$ ) and $\mathbf{w}$ demonstrate that $\mathbf{f}_{i} \subset \mathbf{c}_{i}$, which is a contradiction.

Suppose that $i \in[q+1, r]$. Let $V_{1}\left(F_{i}\right)$ consists of those vertices which are mapped by $h: F_{i} \rightarrow G_{i}$ into $L$ and let $V_{2}\left(F_{i}\right)=V\left(F_{i}\right) \backslash V_{1}\left(F_{i}\right)$. This is a legitimate bipartition of $F_{i}$ because any colour- $i$ edge of $G$ connects $L$ to $V(G) \backslash L$. Let $y \in V_{2}\left(F_{i}\right)$. The sets $C_{B_{1}, \ldots, B_{r}}$ 's partition $V(G) \backslash L$; let $y \in C_{B_{1}, \ldots, B_{r}}$. If $\{y, z\} \in E\left(F_{i}\right), z \in V_{1}\left(F_{i}\right)$, then $B_{i} \ni h(z)$, which, together with $c_{B_{1}, \ldots, B_{r}}>0$ shows that $F_{i} \subset \mathbf{g}_{i}$. This contradiction proves the theorem.

\section{Complete Bipartite Graphs}

Here we will compute asymptotically the size Ramsey number if each forbidden graph is a complete bipartite graph. More precisely, we show that in order to do this it is enough to consider only complete bipartite graphs having the arrowing property.

Theorem 6 Let $r \geq 2$ and $q \geq 1$. Suppose that we are given $t_{1}, \ldots, t_{q} \in \mathbb{R}_{>0}$ and $s_{1}, \ldots, s_{r}, t_{q+1}, \ldots, t_{r} \in \mathbb{N}$ such that $t_{i} \geq s_{i}$ for $i \in[q+1, r]$. Then there exist $s \in \mathbb{N}$ and $t \in \mathbb{R}_{>0}$ such that $\mathbf{k}_{s, t} \rightarrow \mathbf{F}$ and $\hat{r}(\mathbf{F})=e\left(\mathbf{k}_{s, t}\right)=$ st, where

$$
\mathbf{F}=\left(\mathbf{k}_{s_{1}, t_{1}}, \ldots, \mathbf{k}_{s_{q}, t_{q}}, K_{s_{q+1}, t_{q+1}}, \ldots, K_{s_{r}, t_{r}}\right) .
$$

Proof. Let us first describe an algorithm finding extremal $s$ and $t$. Some by-product information gathered by our algorithm will be used in the proof of the extremality of $\mathbf{k}_{s, t} \rightarrow \mathbf{F}$.

Choose $l \in \mathbb{N}$ bigger than $\hat{r}(\mathbf{F}) / t_{0}$, where $t_{0}=\sum_{i=1}^{q} t_{i}$, which is the same definition of $l$ as that before Lemma 3 .

We claim that $l>\sigma$, where $\sigma=\sum_{i=1}^{r}\left(s_{i}-1\right)$. Indeed, take any extremal $\mathbf{f} \rightarrow \mathbf{F}$ without isolated vertices. The proof of Lemma 3 implies that $d(x) \geq t_{0}$ for any $x \in V(\mathbf{f})$. Note that necessarily $v(\mathbf{f})>\sigma$, which implies the claim. 
For each integer $s \in[\sigma+1, l]$, let $t_{s}^{\prime}>0$ be the infimum of $t \in \mathbb{R}$ such that $\mathbf{k}_{s, t} \rightarrow \mathbf{F}$. Also, let $\Pi_{s}$ be the set of all sequences $\mathbf{a}=\left(a_{1}, \ldots, a_{r}\right)$ of non-negative integers with $a_{i}=s_{i}-1$ for $i \in[q+1, r]$ and $\sum_{i=1}^{r} a_{i}=s$. For a sequence $\mathbf{a}=\left(a_{1}, \ldots, a_{r}\right)$ and a set $A$ of size $\sum_{i=1}^{r} a_{i}$, let $\left(\begin{array}{l}A \\ \mathbf{a}\end{array}\right)$ consist of all sequences $\mathbf{A}=\left(A_{1}, \ldots, A_{r}\right)$ of sets partitioning $A$ with $\left|A_{i}\right|=a_{i}, i \in[r]$.

We claim that $t_{s}^{\prime}$ is $\operatorname{sol}\left(L_{s}\right)$, the extremal value of the following linear program $L_{s}$ : "Find $\operatorname{sol}\left(L_{s}\right)=\max \sum_{\mathbf{a} \in \Pi_{s}} w_{\mathbf{a}}$ over all sequences $\left(w_{\mathbf{a}}\right)_{\mathbf{a} \in \Pi_{s}}$ of non-negative reals such that

$$
\sum_{\mathbf{a} \in \Pi_{s}} w_{\mathbf{a}}\left(\begin{array}{c}
a_{i} \\
s_{i}
\end{array}\right) \leq t_{i}\left(\begin{array}{c}
s \\
s_{i}
\end{array}\right), \quad \text { for all } i \in[q] . "
$$

Claim 1 The weight $\mathbf{k}_{s, t}$ does not arrow $\mathbf{F}$ for $t<\operatorname{sol}\left(L_{s}\right)$.

To prove this, let

$$
\lambda=\frac{t+\operatorname{sol}\left(L_{s}\right)}{2 \operatorname{sol}\left(L_{s}\right)}<1 \quad \text { and } \quad \epsilon=\frac{1-\lambda}{2 r^{l}} \min \left\{t_{i} \mid i \in[q]\right\}>0 .
$$

Let $V\left(\mathbf{k}_{s, t}\right)=[s]$. Define an $r$-colouring $\mathbf{c}$ of $\mathbf{k}_{s, t}$ by

$$
\left.c_{\mathbf{A}}=\frac{\lambda w_{\left|A_{1}\right|, \ldots,\left|A_{r}\right|} \mid}{\left(\left|A_{1}\right|, \ldots,\left|A_{r}\right|\right.}\right), \quad \mathbf{a} \in \Pi_{s}, \mathbf{A} \in\left(\begin{array}{c}
{[s]} \\
\mathbf{a}
\end{array}\right),
$$

$c_{B, \emptyset, \ldots, \emptyset}=\epsilon, B \subsetneq[s]$, while all other $c$ 's are zero. It is indeed a colouring:

$$
\sum_{\mathbf{a} \in \Pi_{s}} \sum_{\mathbf{A} \in\left(\begin{array}{c}
{[s]} \\
\mathbf{a}
\end{array}\right)} c_{\mathbf{A}}=\sum_{\mathbf{a} \in \Pi_{s}} \lambda w_{\mathbf{a}}=\lambda \operatorname{sol}\left(L_{s}\right)>t .
$$

We have $\mathbf{k}_{s_{i}, t_{i}} \not \subset \mathbf{c}_{i}$ for $i \in[q]$. For example, for $i=1$ and any $S \in\left(\begin{array}{l}{[s]} \\ s_{1}\end{array}\right)$, we have

$$
\sum_{\mathbf{a} \in \Pi_{s}} \sum_{\substack{\mathbf{A} \in\left(\begin{array}{c}
{[s] \\
\mathbf{a}}
\end{array}\right) \\
A_{1} \supset S}} c_{\mathbf{A}}=\sum_{\substack{\mathbf{a} \in \Pi_{s} \\
a_{1} \geq s_{1}}} \frac{\left(\begin{array}{c}
s-s_{1} \\
a_{1}-s_{1}, a_{2}, \ldots, a_{r}
\end{array}\right) \lambda w_{\mathbf{a}}}{\left(\begin{array}{c}
s, \ldots, a_{r} \\
a_{1}, \ldots
\end{array}\right)}=\lambda \sum_{\mathbf{a} \in \Pi_{s}} \frac{\left(\begin{array}{c}
a_{1} \\
s_{1}
\end{array}\right) w_{\mathbf{a}}}{\left(\begin{array}{c}
s \\
s_{1}
\end{array}\right)} \leq \lambda t_{1}<t_{1}-\sum_{\substack{B \subseteq[s] \\
B \supset S}} c_{B, \emptyset, \ldots, \emptyset .}
$$

Also, $K_{s_{i}, t_{i}} \not \subset \mathbf{c}_{i}$ for $i \in[q+1, r]$ because $c_{A_{1}, \ldots, A_{r}}=0$ whenever $\left|A_{i}\right| \geq s_{i}$ for some $i \in[q+1, r]$. Claim 1 is proved.

Claim $2 \mathbf{k}_{s, t} \rightarrow \mathbf{F}$ for any $t>\operatorname{sol}\left(L_{s}\right)$.

Suppose that the claim is not true and we can find an $\mathbf{F}$-free $r$-colouring $\mathbf{c}$ of $\mathbf{k}_{s, t}$. By the definition, $c_{A_{1}, \ldots, A_{r}}=0$ whenever $\left|A_{i}\right| \geq s_{i}$ for some $i \in[q+1, r]$. If some $c_{A_{1}, \ldots, A_{r}}=c>0$ with $\left|A_{i}\right| \leq s_{i}-2$ for some $i \in[q+1, r]$, then $A_{j} \neq \emptyset$ for some $j \in[q]$, so we can pick

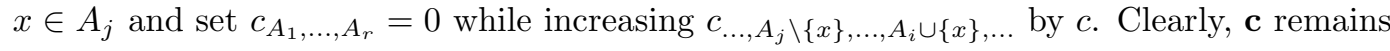
an $\mathbf{F}$-free colouring. Thus, we can assume that all the $c$ 's are zero except those of the form $c_{\mathbf{A}}, \mathbf{A} \in\left(\begin{array}{c}{[s]} \\ \mathbf{a}\end{array}\right)$ for some $\mathbf{a} \in \Pi_{s}$. Now, retracing back our proof of Claim 1, we obtain a feasible solution $w_{\mathbf{a}}=\sum_{\mathbf{A} \in\left(\begin{array}{c}{[s]} \\ \mathbf{a}\end{array}\right)} c_{\mathbf{A}}, \mathbf{a} \in \Pi_{s}$, to $L_{s}$ with a larger objective function, which is a contradiction. The claim is proved.

Thus, $t_{s}^{\prime}=\operatorname{sol}\left(L_{s}\right)$ and $m_{u}=\min \left\{s t_{s}^{\prime} \mid s \in[\sigma+1, l]\right\}$ is an upper bound on $\hat{r}(\mathbf{F})$. Let us show that in fact $\hat{r}(\mathbf{F})=m_{u}$.

We rewrite the definition of $\hat{r}(\mathbf{F})$ so that we can apply the Farkas Lemma. The proof of the following easy claim is left to the reader. 
Claim $3 \hat{r}(\mathbf{F})=\inf e(\mathbf{g})$ over all weights $\mathbf{g}$ on $L$ such that there do not exist non-negative reals $\left(c_{\mathbf{A}}\right)_{\mathbf{A} \in\left(\begin{array}{l}A \\ \mathbf{a}\end{array}\right), \mathbf{a} \in \Pi_{|A|}, A \in 2^{L}}$ with the following properties

$$
\begin{aligned}
& \sum_{\mathbf{a} \in \Pi_{|A|}} \sum_{\mathbf{A} \in\left(\begin{array}{c}
A \\
\mathbf{a}
\end{array}\right)} c_{\mathbf{A}} \geq g_{A}, \quad A \in 2^{L},
\end{aligned}
$$

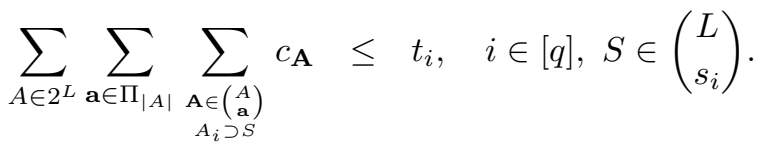

Let $\mathbf{g}$ be any feasible solution to the above problem. By the Farkas Lemma there exist $x_{A} \geq 0, A \in 2^{L}$, and $y_{i, S} \geq 0, i \in[q], S \in\left(\begin{array}{l}L \\ s_{i}\end{array}\right)$, such that

$$
\begin{aligned}
& \sum_{i=1}^{q} \sum_{S \in\left(\begin{array}{l}
A_{i} \\
s_{i}
\end{array}\right)} y_{i, S} \geq x_{A}, \quad A \in 2^{L}, \mathbf{a} \in \Pi_{|A|}, \quad \mathbf{A} \in\left(\begin{array}{l}
A \\
\mathbf{a}
\end{array}\right), \\
& \sum_{i=1}^{q} t_{i} \sum_{S \in\left(\begin{array}{l}
L \\
s_{i}
\end{array}\right)} y_{i, S}<\sum_{A \in 2^{L}} g_{A} x_{A} .
\end{aligned}
$$

We deduce that $x_{A} \leq 0$ (and hence $x_{A}=0$ ) if $|A| \leq \sigma$ by considering (12) for some $\mathbf{A}$ with $\left|A_{i}\right| \leq s_{i}-1$, each $i \in[r]$.

For each $A$ with $a:=|A|>\sigma$ repeat the following. Let $\left(w_{\mathbf{a}}\right)_{\mathbf{a} \in \Pi_{a}}$ be an extremal solution to $L_{a}$. For each $\mathbf{a} \in \Pi_{a}$, take the average of (12) over all $\mathbf{A} \in\left(\begin{array}{l}A \\ \mathbf{a}\end{array}\right)$, multiply it by $w_{\mathbf{a}}$, and add all these equalities together to obtain the following.

$$
\begin{aligned}
x_{A} t_{a}^{\prime} & =\sum_{\mathbf{a} \in \Pi_{a}} \sum_{\mathbf{A} \in\left(\begin{array}{c}
A \\
\mathbf{a}
\end{array}\right)} w_{\mathbf{A}} x_{A} \leq \sum_{\mathbf{a} \in \Pi_{a}} \frac{w_{\mathbf{a}}}{\left(\begin{array}{c}
a \\
a_{1}, \ldots, a_{r}
\end{array}\right)} \sum_{\mathbf{A} \in\left(\begin{array}{c}
A \\
\mathbf{a}
\end{array}\right)} \sum_{i=1}^{q} \sum_{S \in\left(\begin{array}{l}
A_{i} \\
s_{i}
\end{array}\right)} y_{i, S} \\
& =\sum_{i=1}^{q} \sum_{S \in\left(\begin{array}{c}
A \\
s_{i}
\end{array}\right)} y_{i, S} \sum_{\substack{\mathbf{a} \in \Pi_{a} \\
a_{i} x_{i}}} \frac{w_{\mathbf{a}}\left(\begin{array}{c}
a-s_{i} \\
a_{1}, \ldots, a_{i-1}, a_{i}-s_{i}, a_{i+1}, \ldots, a_{q}
\end{array}\right)}{\left(\begin{array}{c}
a \\
a_{1}, \ldots, a_{r}
\end{array}\right)} \\
& =\sum_{i=1}^{q} \sum_{S \in\left(\begin{array}{l}
A \\
s_{i}
\end{array}\right)} y_{i, S} \sum_{\substack{\mathbf{a} \in \Pi_{a} \\
a_{i} \geq s_{i}}} \frac{w_{\mathbf{a}}\left(\begin{array}{c}
a_{i} \\
s_{i}
\end{array}\right)}{\left(\begin{array}{c}
a \\
s_{i}
\end{array}\right)} \leq \sum_{i=1}^{q} t_{i} \sum_{S \in\left(\begin{array}{l}
A \\
s_{i}
\end{array}\right)} y_{i, S} .
\end{aligned}
$$

(In the last inequality we used (11).)

Substituting the obtained inequalities on the $x_{A}$ 's into (13) we obtain

$$
\sum_{i=1}^{q} t_{i} \sum_{S \in\left(\begin{array}{c}
L \\
s_{i}
\end{array}\right)} y_{i, S}<\sum_{\substack{A \in 2 L \\
|A|>\sigma}} \frac{g_{A}}{t_{s}^{\prime}} \sum_{i=1}^{q} t_{i} \sum_{S \in\left(\begin{array}{c}
A \\
s_{i}
\end{array}\right)} y_{i, S} .
$$

As the $y_{i, S}$ 's are non-negative, some of these variables has a larger coefficient on the righthand side. Let it be $y_{i, S}$. We have

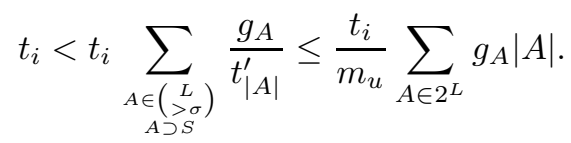

The last inequality follows from the fact that for any integer $a>\sigma$, we have $1 / t_{a}^{\prime} \leq a / m_{u}$, which in turn follows from the definition of $m_{u}$. Hence, $e(\mathbf{g}) \geq m_{u}$ as required. 
Corollary 7 Let $r \geq q \geq 1, t_{1}, \ldots, t_{q} \in \mathbb{R}_{>0}$ and $s_{1}, \ldots, s_{r}, t_{q+1}, \ldots, t_{r} \in \mathbb{N}$ such that $t_{i} \geq s_{i}$ for $i \in[q+1, r]$. For $i \in[q]$, let $\left(t_{i, n}\right)_{n \in \mathbb{N}}$ be an integer sequence with $t_{i, n}=t_{i} n+o(n)$. Define

$$
\mathbf{F}_{n}=\left(K_{s_{1}, t_{1, n}}, \ldots, K_{s_{q}, t_{q, n}}, K_{s_{q+1}, t_{q+1}}, \ldots, K_{s_{r}, t_{r}}\right) .
$$

Let $l \in \mathbb{N}$ be larger than $\lim _{n \rightarrow \infty} \hat{r}\left(\mathbf{F}_{n}\right) / t_{0} n$, where $t_{0}=\sum_{i=1}^{q} t_{i}$. Then

$$
\lim _{n \rightarrow \infty} \frac{\hat{r}\left(\mathbf{F}_{n}\right)}{n}=\lim _{n \rightarrow \infty} \frac{\min \left\{e\left(K_{s, t}\right) \mid s \leq l, K_{s, t} \rightarrow \mathbf{F}_{n}\right\}}{n} .
$$

In other words, in order to compute the limit in Corollary 7 it is sufficient to consider only complete bipartite graphs arrowing $\mathbf{F}_{n}$. It seems that there is no simple general formula, but the proof of Theorem 6 gives an algorithm for computing $\hat{r}(\mathbf{F})$. The author has realized the algorithm as a C program calling the lp_solve library. (The latter is a freely available linear programming software, currently maintained by Michel Berkelaar [5]). Later, David Avis rewrote the program to be linked with his lrslib library [i]. The latter library has the advantage that its arithmetic is exact (whilst lp_solve operates with reals), so that any computed limit can be considered as proved (provided the realisation of our algorithm is correct). The reader is welcome to experiment with the program; its source is included at the end of this paper.

For certain series of parameters we can get a more explicit expression. First, let us treat the case $q=1$, that is, when only the first forbidden graph dilates with $n$. We can assume that $t_{1}=1$ by scaling $n$.

Theorem 8 Let $q=1$ and $r \geq 2$. Then for any $s_{1}, \ldots, s_{r}, t_{2}, \ldots, t_{r} \in \mathbb{N}$ with $t_{i} \geq s_{i}$, $i \in[2, r]$, we have

$$
\hat{r}\left(K_{s_{1}, n}, K_{s_{2}, t_{2}}, \ldots, K_{s_{r}, t_{r}}\right)=n \cdot \min \left\{s \frac{(s)_{s_{1}}}{\left(s-s^{\prime}\right)_{s_{1}}} \mid s \in \mathbb{N}_{>\sigma}\right\}+O(1),
$$

where $s^{\prime}=\sigma-s_{1}+1, \sigma=\sum_{i=1}^{r}\left(s_{i}-1\right)$ and $(s)_{k}=s(s-1) \ldots(s-k+1)$.

Proof. The Problem $L_{s}$ has only one variable $w_{s-s^{\prime}, s_{2}-1, \ldots, s_{r}-1}$. Trivially, $t_{s}^{\prime}=\left(\begin{array}{c}s \\ s_{1}\end{array}\right) /\left(\begin{array}{c}s-s^{\prime} \\ s_{1}\end{array}\right)=$ $(s)_{s_{1}} /\left(s-s^{\prime}\right)_{s_{1}}$, and the theorem follows.

In the case $s_{1}=1$ we obtain the following formula (with a little bit of algebra).

Corollary 9 For any $s_{2}, \ldots, s_{r}, t_{2}, \ldots, t_{r} \in \mathbb{N}$ with $t_{i} \geq s_{i}, i \in[2, r]$, we have

$$
\hat{r}\left(K_{1, n}, K_{s_{2}, t_{2}}, \ldots, K_{s_{r}, t_{r}}\right)=4\left(1-r+\sum_{i=2}^{r} s_{i}\right) n+O(1) .
$$

Another case with a simple formula for $\hat{r}(\mathbf{F})$ is $q=2, s_{1}=s_{2}$, and $t_{1}=t_{2}$. Again, without loss of generality we can assume that $t_{1}=t_{2}=1$.

Theorem 10 Let $q=2$ and $r \geq 2$. Then for any $s, s_{3}, \ldots, s_{r}, t_{3}, \ldots, t_{r} \in \mathbb{N}$ with $t_{i} \geq s_{i}$, $i \in[3, r]$, we have

$$
\hat{r}\left(K_{s, n}, K_{s, n}, K_{s_{3}, t_{3}}, \ldots, K_{s_{r}, t_{r}}\right)=n \cdot \min \left\{a \cdot f(a) \mid a \in \mathbb{N}_{>\sigma}\right\}+O(1),
$$


where $\sigma=2 s-r+\sum_{i=3}^{r} s_{i}$ and

$$
f(a)=\frac{2\left(\begin{array}{l}
a \\
s
\end{array}\right)}{\left(\begin{array}{c}
\left\lfloor a^{\prime} / 2\right\rfloor \\
s
\end{array}\right)+\left(\begin{array}{c}
\left\lceil a^{\prime} / 2\right\rceil \\
s
\end{array}\right)},
$$

with $a^{\prime}=a-\sum_{i=3}^{r}\left(s_{i}-1\right)$.

Proof. Let $a \in \mathbb{N}_{>\sigma}$ and let $\left(w_{\mathbf{a}}\right)_{\mathbf{a} \in \Pi_{a}}$ be an extremal solution to $L_{a}$. (Where we obviously define $s_{1}=s_{2}=s$ and $t_{1}=t_{2}=1$.) Excluding the constant indices in $w_{\mathbf{a}}$, we assume that the index set $\Pi_{a}$ consists of pairs of integers $\left(a_{1}, a_{2}\right)$ with $a_{1}+a_{2}=a^{\prime}$.

Clearly, $w_{a_{1}, a_{2}}^{\prime}=\frac{1}{2}\left(w_{a_{1}, a_{2}}+w_{a_{2}, a_{1}}\right),\left(a_{1}, a_{2}\right) \in \Pi_{a}$, is also an extremal solution, so we can assume that $w_{a_{1}, a_{2}}=w_{a_{2}, a_{1}}$ for all $\left(a_{1}, a_{2}\right) \in \Pi_{a}$.

If $w_{a_{1}, a_{2}}=c>0$ for some $a_{1}<\left\lfloor a^{\prime} / 2\right\rfloor$, then we can set $w_{a_{1}, a_{2}}=w_{a_{2}, a_{1}}=0$, while increasing $w_{\left\lfloor a^{\prime} / 2\right\rfloor,\left\lceil a^{\prime} / 2\right\rceil}$ and $w_{\left\lceil a^{\prime} / 2\right\rceil,\left\lfloor a^{\prime} / 2\right\rfloor}$ by $c$. The easy inequality

$\left(\begin{array}{c}b+1 \\ s\end{array}\right)+\left(\begin{array}{c}a^{\prime}-b-1 \\ s\end{array}\right)-\left(\begin{array}{l}b \\ s\end{array}\right)-\left(\begin{array}{c}a^{\prime}-b \\ s\end{array}\right)=\left(\begin{array}{c}b \\ s-1\end{array}\right)-\left(\begin{array}{c}a^{\prime}-b-1 \\ s-1\end{array}\right)<0, \quad s-1 \leq b<\left\lfloor a^{\prime} / 2\right\rfloor$

implies inductively that the left-hand side of (11) strictly decreases while the objective function $\sum_{\mathbf{a} \in \Pi_{a}} w_{\mathbf{a}}$ does not change, which clearly contradicts the minimality of $\mathbf{w}$.

Now we deduce that, for any extremal solution $\left(w_{\mathbf{a}}\right)_{\mathbf{a} \in \Pi_{a}}$, we have $w_{a_{1}, a_{2}}=0$ unless $\left\{a_{1}, a_{2}\right\}=\left\{\left\lfloor a^{\prime} / 2\right\rfloor,\left\lceil a^{\prime} / 2\right\rceil\right\}$; moreover, it follows now that necessarily $w_{\left\lfloor a^{\prime} / 2\right\rfloor,\left\lceil a^{\prime} / 2\right\rceil}=$ $w_{\left\lceil a^{\prime} / 2\right\rceil,\left\lfloor a^{\prime} / 2\right\rfloor}$. Hence, $t_{a}^{\prime}=f(a)$, which proves the theorem.

The special case $r=2$ of Theorem 10 answers the question of Erdös, Faudree, Rousseau and Schelp [10, Problem B], who asked for the asymptotics of $\hat{r}\left(K_{s, n}, K_{s, n}\right)$. Unfortunately, we do not think that the formula (16) can be simplified further in this case.

Finally, let us consider the case $(r, s)=(2,2)$ of Theorem 10 in more detail. It is routine to check that Theorem 10 implies that $\hat{r}\left(K_{2, n}, K_{2, n}\right)=18 n+O(1)$. But we are able to show that (3) holds for all sufficiently large $n$, which is done by showing that $\left(K_{2, n}, K_{2, n}\right)$-arrowing graph with $18 n+o(n)$ edges can have at most 3 vertices of degree at least $n$ for all large $n$.

Theorem 11 There is $n_{0}$ such that, for all $n>n_{0}$, we have $\hat{r}\left(K_{2, n}, K_{2, n}\right)=18 n-15$ and $K_{3,6 n-5}$ is the only extremal graph (up to isolated vertices).

Proof. For $n \in \mathbb{N}$ let $G_{n}$ be a minimum $\left(K_{2, n}, K_{2, n}\right)$-arrowing graph. We know that $e\left(G_{n}\right) \leq 18 n-15$ so $l_{n}=\left|L_{n}\right| \leq 18$ for all large $n$, where $L_{n}=\left\{x \in V\left(G_{n}\right) \mid d(x) \geq n\right\} ;$ let us assume $L_{n} \subset[18]$.

Claim $1 l_{n} \leq 3$ for all sufficiently large $n$.

Suppose on the contrary that we can find an increasing subsequence $\left(n_{i}\right)_{i \in \mathbb{N}}$ with $l_{n_{i}} \geq 4$ for all $i$. Choosing a further subsequence, assume that $L_{n_{i}}=L$ does not depend on $i$ and that $g_{A}=\lim _{i \rightarrow \infty}\left|G_{n_{i}}^{A}\right| / n_{i}$ exists for any $A \in 2^{L}$. The argument of Lemma 1 shows that the weight $\mathbf{g}$ on $L$ arrows $\left(\mathbf{k}_{2,1}, \mathbf{k}_{2,1}\right)$.

We have $e(\mathbf{g})=18$. It is routine to check that $a t_{a}^{\prime}>18$ for any $a \in[4,18]$. The inequality (14) implies that, for some $S=\{x, y\} \subset L$, we have $g_{A}=0$ whenever $|A|>4$ or $A \not \supset S$. Let $J$ be the set of those $j \in L$ with $g_{\{x, y, j\}}>0$. We have $\sum_{j \in J} g_{\{x, y, j\}}=6$. 
Consider the 2-colouring $\mathbf{c}$ of $\mathbf{g}$ obtained by letting $c_{A_{1}, A_{2}}=2^{-18} / 10$ for all disjoint $A_{1}, A_{1} \in 2^{L}$ except

$$
\begin{aligned}
& c_{\{x, j\},\{y\}}=c_{\{y, j\},\{x\}}=c_{\{x\},\{y, i\}}=c_{\{y\},\{x, i\}}=0.9, \quad j \in J, \\
& c_{\{x, y\},\{j\}}=c_{\{j\},\{x, y\}}=\left(g_{\{x, y, j\}}-3.5\right)_{+} / 2,
\end{aligned}
$$

where $f_{+}=f$ if $f>0$ and $f_{+}=0$ if $f \leq 0$. It is easy to check that neither $\mathbf{c}_{1}$ nor $\mathbf{c}_{2}$ contains $\mathbf{k}_{2,1}$ : for example, $\sum_{A \in 2^{L}: A \supset\{x, y\}} c_{i, A}<(5-3.5) / 2+0.1<1$. (Recall that $d_{\mathbf{g}}(x) \geq 1$ for all $x \in L$.) This contradiction proves Claim 1 .

Thus, $\left|L_{n}\right| \leq 3$ for all large $n$. By the minimality of $G_{n}, V\left(G_{n}\right) \backslash L_{n}$ spans no edge and each $x \in V\left(G_{n}\right) \backslash L_{n}$ sends at least 3 edges to $L_{n}$. (In particular, $\left|L_{n}\right|=3$.) Thus, disregarding isolated vertices, $G_{n}=K_{3, m}$. The relation $G_{n} \rightarrow\left(K_{2, n}, K_{2, n}\right)$ implies that $m \geq 6 n-5$, which proves the theorem.

Remark. We do not write an explicit expression for $n_{0}$, although it should be possible to extract this from the proof (with more algebraic work) by using the estimates of Theorem 5 .

\section{$5 \quad$ Generalizations}

If all forbidden graphs are the same, then one can generalize the arrowing property in the following way: a graph $G(r, s)$-arrows $F$ if for any $r$-colouring of $E(G)$ there is an $F$ subgraph that receives less than $s$ colours. Clearly, in the case $s=2$ we obtain the usual $r$-colour arrowing property $G \rightarrow(F, \ldots, F)$.

This property was first studied by Ekeles, Erdős and Füredi (as reported in [8, Section 9]); the reader can consult [2] for references to more recent results.

Axenovich, Füredi and Mubayi [2] studied the generalized arrowing property for bipartite graphs in the situation when $F$ and $s$ are fixed, $G=K_{n, n}$, and $r$ grows with $n$.

We can define $\hat{r}(F, r, s)$ to be the minimal size of a graph which $(r, s)$-arrows $F$. Our technique extends to the case when $r$ and $s$ are fixed whilst $F$ grows with $n$ (i.e., is a dilatation). Namely, it should be possible to show the following.

Let $\left(F_{n}\right)_{n \in \mathbb{N}}$ be a dilatation of a weight $\mathbf{f}$ and let $r \geq s$ be fixed. Then the limit $\lim _{n \rightarrow \infty} \hat{r}\left(F_{n}, r, s\right) / n$ exists; let us denote it by $\hat{r}(\mathbf{f}, r, s)$.

We have $\hat{r}(\mathbf{f}, r, s)<\infty$ and, in fact, $\hat{r}(\mathbf{f}, r, s)=\min e(\mathbf{g})$ over all weights $\mathbf{g}$ such that for any $r$-colouring $\mathbf{c}$ of $\mathbf{g}$ there is $S=\left\{i_{1}, \ldots, i_{s}\right\} \in\left(\begin{array}{c}{[r]} \\ s\end{array}\right)$ such that $\mathbf{c}_{S} \supset \mathbf{f}$, where

$$
c_{S, A}=\sum_{A_{1}, \ldots, A_{r}} c_{A_{1}, \ldots, A_{r}}, \quad A \in 2^{V(\mathbf{g})}
$$

where the sum is taken over all disjoint $A_{1}, \ldots, A_{r} \in 2^{L}$ with $A_{i_{1}} \cup \ldots \cup A_{i_{s}}=A$.

We omit the proof as the complete argument would not be very short and it is fairly obvious how to proceed.

Also, one can consider the following settings. Let $\mathcal{F}_{i}$ be a family of graphs, $i \in[r]$. We write $G \rightarrow\left(\mathcal{F}_{1}, \ldots, \mathcal{F}_{r}\right)$ if for any $r$-colouring of $E(G)$, there is $i \in[r]$ and $F \in \mathcal{F}_{i}$ such that we have an $F$-subgraph of colour $i$. The task is to compute the minimum size of a such $G$. Again, we believe that our method extends to this case as well. But we do not provide any proof, so we do not present this as a theorem. 


\section{Acknowledgements}

I am grateful to Martin Henk, Deryk Osthus, and Günter Ziegler for helpful discussions and to David Avis for rewritting and improving my code (and for his nice lrslib library!).

\section{References}

[1] D. Avis, lrs, http://cgm.cs.mcgill.ca/ªvis/C/lrs.html.

[2] M. Axenovich, Z. Füredi, and D. Mubayi, On generalized Ramsey theory: The bipartite case, J. Combin. Theory (B) 79 (2000), 66-86.

[3] J. Beck, On size Ramsey number of pathes, trees, and circuits, I, J. Graph Theory 7 (1983), 115-129.

[4] - On size Ramsey number of stars, trees, and circuits, II, Mathematics of Ramsey Theory (J. Nešetril and V. Rödl, eds.), Springer, Berlin, 1990, pp. 34-45.

[5] M. Berkelaar, lp_solve, ftp://ftp.ics.ele.tue.nl/pub/lp_solve/.

[6] S. A. Burr, P. Erdős, R. J. Faudree, C. C. Rousseau, and R. H. Schelp, Ramsey-minimal graphs for multiple copies, Indag. Math. 40 (1978), 187-195.

[7] P. Erdős, Problems and results in graph theory, The Theory and Applications of Graphs (G. Chartrand, ed.), John Wiley, New York, 1981, pp. 331-341.

[8] _ Solved and unsolved problems in combinatorics and combinatorial number theory, Congres. Numer. 32 (1981), 49-62.

[9] P. Erdős and R. J. Faudree, Size Ramsey functions, Sets, graphs and numbers (Budapest, 1991), North-Holland, Amsterdam, 1992, pp. 219-238.

[10] P. Erdős, R. J. Faudree, C. C. Rousseau, and R. H. Schelp, The size Ramsey number, Period. Math. Hung. 9 (1978), 145-161.

[11] P. Erdős and C. C. Rousseau, The size Ramsey number of a complete bipartite graph, Discrete Math. 113 (1993), 259-262.

[12] R. J. Faudree, C. C. Rousseau, and J. Sheehan, A class of size Ramsey problems involving stars, Graph Theory and Combinatorics, Proc. Conf. Hon. P. Erdős, Cambridge (B. Bollobas, ed.), Cambridge Univ. Press, 1983, pp. 273-281.

[13] P. E. Haxell and Y. Kohayakawa, The size-Ramsey number of trees, Israel J. Math. 89 (1995), 261-274.

[14] X. Ke, The size Ramsey number of trees with bounded degree, Random Struct. Algorithms 4 (1993), 85-97.

[15] R. Lortz and I. Mengersen, Size Ramsey results for paths versus stars, Australas. J. Comb. 18 (1998), 3-12. 
[16] O. Pikhurko, Size Ramsey numbers of stars versus 3-chromatic graphs, Combinatorica, 21 (2001) 403-412.

[17] _ Size Ramsey numbers of stars versus 4-chromatic graphs, Submitted to J. Graph Theory, 2001. 


\section{C source code}

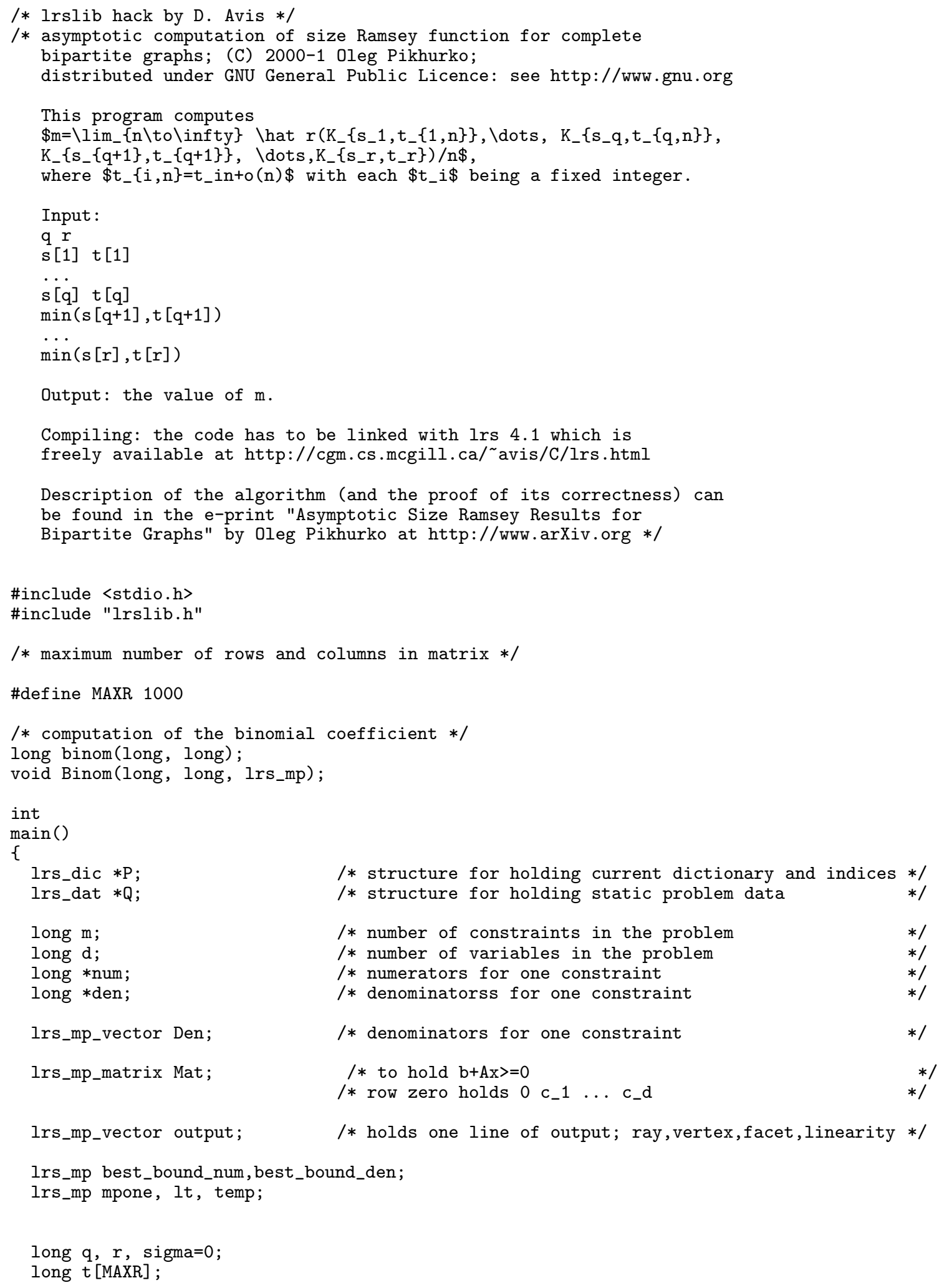









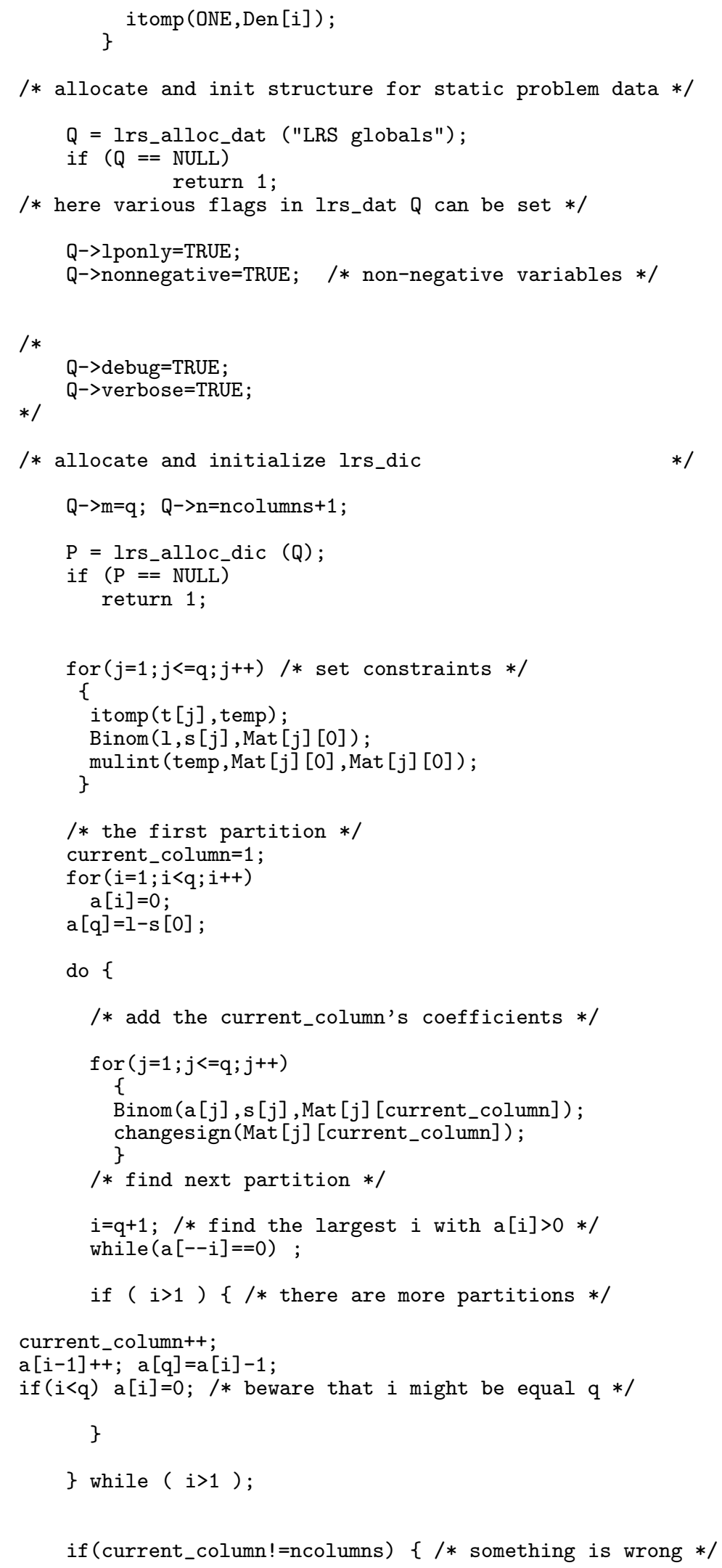




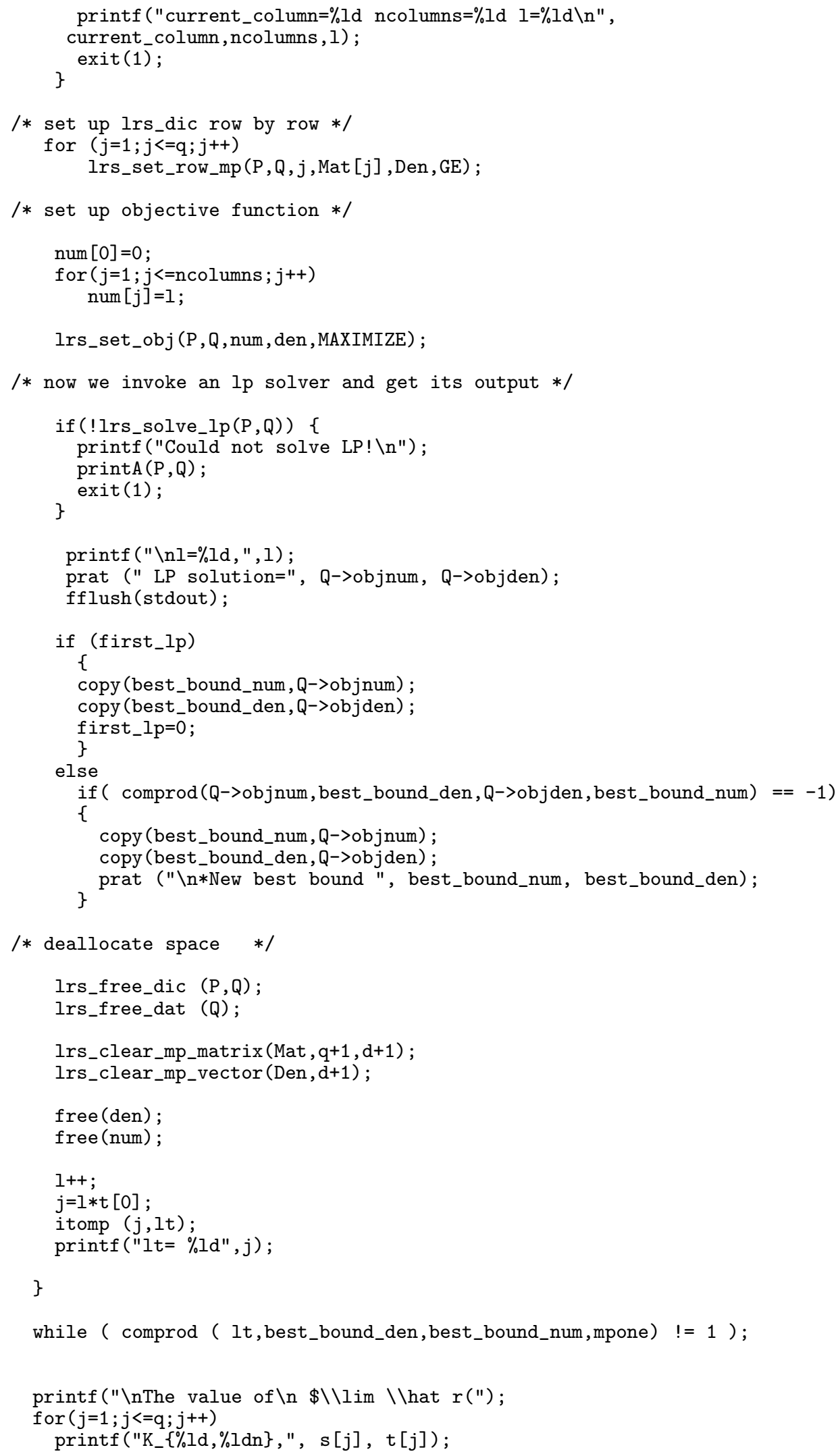




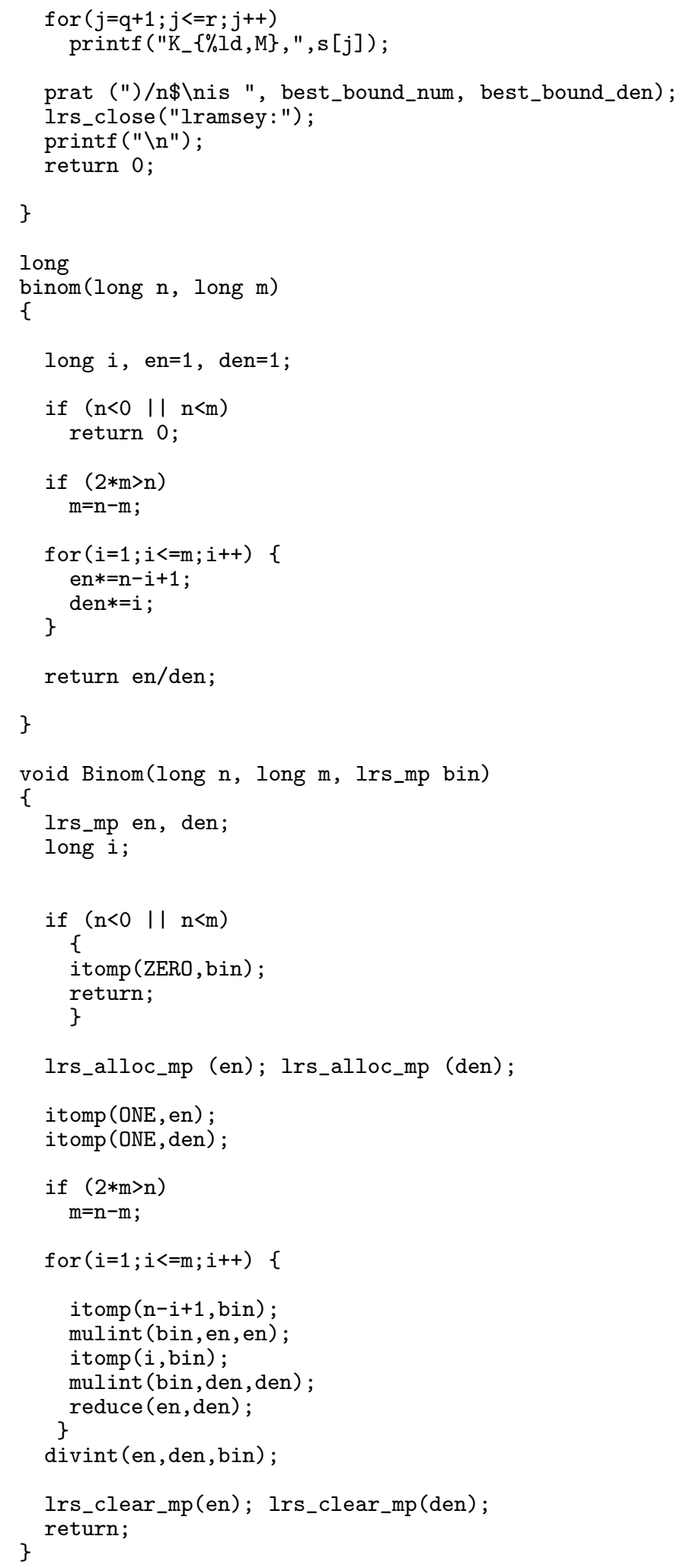

